\title{
Enteral diclofenac controls pain and reduces intravenous injection during extracorporeal shock wave lithotripsy
}

\author{
Mehrdad Mesbah Kiaei (1), Mahmoud Reza Mohaghegh (2), Gholamreza Movaseghi \\ (2), Masoud Ghorbanlo (1) \\ (1) Hasheminejad Kidney Center, School of Medicine, Iran University of Medical Sciences, \\ Tehran, Iran; (2) Department of Anesthesia and Critical Care, Hasheminejad Kidney Center, \\ School of Medicine, Iran University of Medical Sciences, Tehran, Iran
}

This article is distributed under the terms of the Creative Commons Attribution Noncommercial License (CC BY-NC 4.0) which permits any noncommercial use, distribution, and reproduction in any medium, provided the original author(s) and source are credited.

\begin{abstract}
Urinary system stones are the third most common disease of urinary system following urinary infection and prostate pathology. Extracorporeal shockwave lithotripsy (ESWL) is one of the methods used to treat Urolithiasis where shockwaves are transmitted through skin and body organs and crush the stones into small pieces. This is a painful procedure which usually requires analgesics. Each analgesic drug has its own advantages and disadvantages. The present research seeks to study the effectiveness of using diclofenac suppository in order to control pain and reduce need for venous drugs during ESWL procedure. This is a double blind clinical trial. 158 patients resorting to Shahid Hasheminezhad Specialized Center for ESWL were randomly selected to take part in this projects with due consideration of inclusion criteria. The patients were equally divided into the $S$ (diclofenac suppository) and A (placebo) groups. 2 diclofenac suppositories were used in the $\mathrm{S}$ group 20 minutes prior to beginning ESWL. Then, ESWL was carried out in supine position using fluoroscopic conduction with standard method. The present research has studied pain scale of patients, operator's and patient's satisfaction during the operation and patient's hemodynamic parameters in three phases prior to, during, and after ESWL. SPSS v.17 was used to study the data and Chi-Square Tests and Repeated Measure ANOVA were used to analyze the results. The level of significance in the present research was set to $\mathrm{P}$-value $<0.05$. A review of pain scales across both groups showed that using diclofenac suppository has a significant influence in reducing the pain scale and, hence, need for venous drugs $(\mathrm{P}$-value $<0.05)$. No significant difference was observed between the two groups in terms of heart beat and blood pressure changes in various times $(\mathrm{P}$-value $>$ 0.05). The results also report different satisfaction levels for patients and operators across the two groups (diclofenac suppository and placebo) (P-value $<0.05$ ). Higher satisfaction levels were observed among both patients and operators in the group receiving diclofenac suppository. A general look at the data yields the conclusion that receiving pain killers (diclofenac suppository) before starting ESWL plays a more efficient role in reducing pain scales of patients and enhancing operators' satisfaction..
\end{abstract}

Key Words: Extracorporeal shock wave lithotripsy, ESWL, Diclofenac, suppository, pain reduction

Eur J Transl Myol 28 (2): 210-214, 2018

Urinary system stones are the third most common urinary system disease following urinary infection and prostate pathology. ${ }^{1,2}$ Urolithiasis is a common complaint with which people usually resort to medical clinics. A reduction in the number of inhibiting factors (Phytate) in urine and thick urine are two main causes of stone formation. Urinary system stones have other symptoms such as blood in urine, frequent urination and dysuria. However, some cases of Urolithiasis have no symptoms. ${ }^{3}$ The prevalence of Urolithiasis in whole life of people has been estimated around 1 to $15 \%$ varying based upon age, gender, race, and geographical location. The prevalence rate of this disease among men is 2 to 3 times more than what is observed among women. The 


\section{Diclofenac for shock wave lithotripsy}

Eur J Transl Myol 28 (2): 210-214, 2018

most common types of Urolithiasis include Calcium oxalate, calcium phosphate, ammonium-magnesium phosphate, uric acid, and Cystine stones. Lithotripsy is one of the most common methods used to treat kidney stone with little side effects for patients. ${ }^{4}$ As a result of technological developments and availability of various lithotripsy methods such as extracorporeal shockwave lithotripsy (ESWL), Trans-urethral Lithotripsy, and percutaneous Nephrolithotripsy, major steps have been taken in treatment of urinary tract stones. The present research seeks to study the effectiveness of using diclofenac suppository in order to control pain and reduce need for venous drugs during ESWL procedure in Shahid Hasheminezhad specialized center in 2014. ESWL is one of the methods used to treat Urolithiasis where shockwaves are transmitted through skin and body organs and crush the stones into small pieces. A few weeks following this procedure, these crushed pieces would get out of our body through ureter and bladder. ESWL is usually the primary selected therapeutic method to treat kidney stones smaller than $1 \mathrm{~cm}$ and proximal ureter stones. Ureteroscopy and ESWL are the primary treatments for lower ureter stones $^{5,6}$. A research conducted in this field has reported a success rate of 85.3 for ESWL method ${ }^{7}$. Most papers have reported relatively high success rates for extracorporeal shockwave lithotripsy and intracorporeal lithotripsy. ${ }^{8}$ Extracorporeal shockwave lithotripsy is a great revolution in treating urinary tract stones. First, someone in Russia in 1950s came up with the idea of using waves to crush stones. The progresses made in 1980 persuaded researcher to use this method to treat urinary tract stones. Considering the high prevalence rate of urinary tract stones and high expenses of treating these stones through surgery, ESWL can be used as an effective and safe method to treat a large portion of lower and upper urinary tract stones. This method would greatly reduce the need for open surgeries in this field. ${ }^{9,10}$. As a matter of fact, ESWL is the selected therapeutic method for 80 to $90 \%$ of all cases of urinary tract stones. ${ }^{11,12}$

First generation devices such as Dornier HM3 (Human Model 3) were really painful and general or spinal anesthesia was required to treat patients. The second generation of devices made it possible to treat patients under analgesics, but $95 \%$ of patients still experienced pain under the second generation of Dornier HM4 and this pain were reported among $30 \%$ of all cases who didn't use analgesics in third generation devices. This pain would disrupt patient's cooperation and cause intentional or unintentional movements or greater respiratory moves during the procedure. These interventions would disrupt concentration on stone's localized position and reduce shockwave and crushing power. In this method, patient's cooperation is an essential factor which can enhance treatment and crushing quality. As a result, the possibility of stone clearance would decrease significantly. Thus, pain control is an essential factor in treating ESWL patients. In most cases, it is necessary to use pain killers to control this pain including various analgesics including Opioids such as morphine, pethidine, fentanyl, Ketorolac, and codeine which have their own advantages and disadvantages. Treatments using a mixture of oral or injected non-steroid, antiinflammatory drugs along with EMLA cream and Dimethyl Sulfoxide are of high efficiency and little side effects. This therapeutic method requires sufficient analgesia and sedation along with quick recovery, particularly for outpatients. The most effective analgesic protocol needs to be easy, highly effective and with least side effects. As of now, there is no single standard analgesic protocol for these patients and various drugs and protocols are utilized. Opioids are really good analgesics that suppress the pain and enhance patient's satisfaction. However, they have certain complications that elongate the period of patient's monitoring and delay his discharge. Using NSAID's such as diclofenac suppository is easy for both the patient and surgeon. They are easily prescribed and the patient is discharged immediately following the procedure. Drugs such as Paracetamol have a large utilization indication and much attention has been paid to them recently as they have little complications. As a result, the present research seeks to study the effectiveness of using diclofenac suppository in controlling pain and need for less venous painkillers during ESWL and. We will also have a look at patients' hemodynamic parameters and operator's satisfaction across diclofenac suppository and placebo groups.

\section{Materials and Methods}

This is a double-blind clinical trial carried out through randomized sampling. Dornier HM-3 was used for shockwave lithotripsy. The patients were informed of the research and their consents were obtained. As many as 158 patients candidated for ESWL resorting to Shahid Hasheminezhad Hospital took part in the research. The following inclusion criteria were defined: age 20 to 60 years old, class I and II ASA, applying for outpatient ESWL, and kidney stone at the entrance of upper ureter with a diameter of 6 to $20 \mathrm{~mm}$. However, being allergic to drugs, treatment using anti-depression, hypnotic, and Psychedelic drugs, injection or chronic consumption of painkillers or alcohol or using them 3 days prior to the procedure, patients' disagreement to take part in the research, symptoms or signs of patient's urinary tract infections, mild to severe Hydronephrosis, existence of multiple stones, bladder stone, Radiolucent stones, and a ban on using narcotic drugs or neurological diseases. Patients demographic information including their age, weight, gender, and ASA class was recorded using special questionnaires. The patients were divided into class I and II ASA. Class I ASA represents completely healthy and normal patients (class I), while class II ASA represents patients 
Table 1. Pain scale based upon the type of medicine and the statistical correlation between these two parameters among participating patients

\begin{tabular}{|c|c|c|c|c|c|}
\hline \multirow{2}{*}{ P-Value } & \multirow{2}{*}{ Total } & \multicolumn{2}{|c|}{ Drug Groups } & \multicolumn{2}{c|}{ Variable } \\
\cline { 3 - 5 } & & Placebo & Diclofenac Suppository & Low & \multirow{2}{*}{ Pain } \\
\hline \multirow{3}{*}{0.0001} & 51 & 6 & 45 & Mediocre & intensity \\
\cline { 2 - 5 } & 72 & 41 & 31 & High & \\
\cline { 2 - 6 } & 35 & 32 & 3 & \multicolumn{2}{c|}{ Total } \\
\hline \multicolumn{2}{|c|}{158} & 79 & 79 & \multicolumn{2}{c}{} \\
\hline
\end{tabular}

with mild systematic diseases (Class II). The patients were randomly divided into Sh (diclofenac suppository) and A (placebo) groups. 2 diclofenac suppositories were used in the $\mathrm{S}$ group 20 minutes prior to beginning ESWL. Then, ESWL was carried out in supine position using fluoroscopic conduction with standard method. If the pain were too much for the patient to tolerate, they would be given another dose of the medicine. The present research has studied pain scale of patients, operator's and patient's satisfaction during the operation and patient's hemodynamic parameters in three phases prior to, during, and after ESWL. Visual analog scale (VAS) was used to determine the pain score (mild pain (1-3), moderate pain (4-6), and severe pain (7-10)). Then, ESWL was carried out in supine position using fluoroscopic conduction with standard method. If the pain were too much for the patients to tolerate, they would be given another dose of the medicine and as many as 5000 shocks were given. Assessing the individual's satisfaction with situation was achieved by measuring his personal perceptions and attitudes assessed using emotions scales such as Likert index. A 4-degree Likert scale was used in this research to measure the operator's and patient's satisfaction: $1=$ low satisfaction, $2=$ average, $3=$ good, $4=$ excellent. Patients' primary information, pain score, operator's and patient's satisfaction, and complications were recorded in a special form. Having collected the information from ESWL patients, the information was fed to SPSS v.17 and Chi-Square Tests, and Repeated Measure ANOVA were used to analyze the results. The level of significance was set to P-Value $<0.05$.

\section{Results and Discussion}

We selected variables and collected raw data from 158 patients admitted to Shahid Hasheminezhad Specialized Center. Of them, 120 were males $(75.9 \%)$ and 38 females $(24.1 \%)$, aged 21-64 years with an average age of $44.67 \pm 1.13$. The participants weighed 49-100 kilograms with an average weight of $74.29 \pm 0.92$. Based upon non-parametric Table 1 shows a statistically significant difference between the placebo and Diclofenac treated groups in terms of the pain they had felt (Chi-Square Test, P-value < 0.001). Only 3 in diclofenac suppository group, indeed had felt pain during the ESWL procedure and had required intravenous drug injection, while the number in placebo group was 32. Table 2 presents patients' and operators' satisfaction levels in enteral diclofenac and placebo groups analyzed by non-parametric Chi-square test was used to study. Results show significant correlation between patients' and operators' satisfaction and drug consumption (P-value < 0.05). The level of satisfaction for patients and operators in diclofenac suppository group was $86 \%$ and $67 \%$, while the satisfaction levels in the placebo group were $55 \%$ and $34 \%$, respectively. We also monitored in the two groups the effect of either the diclofenac suppository or the placebo on patients' heart rate and systolic - diastolic blood pressures. Table 3 do not show statistically significant differences between the two groups in terms of changes in heart rate and blood pressure prior to, during, and at the end of ESWL (Repeated Measure ANOVA , P-value > 0.05). As a result of technological developments and availability of

Table 2. Operator's level of satisfaction based upon the type of medicine and the statistical correlation between these two parameters among patients

\begin{tabular}{|c|c|c|c|c|}
\hline \multirow[t]{2}{*}{ P-Value } & \multicolumn{2}{|c|}{ Drug Groups } & \multirow{2}{*}{\multicolumn{2}{|c|}{ Variable }} \\
\hline & Placebo & Diclofenac Suppository & & \\
\hline \multirow{2}{*}{0.000} & 44 & 68 & Yes & \multirow{2}{*}{$\begin{array}{c}\text { operator } \\
\text { satisfaction }\end{array}$} \\
\hline & 35 & 11 & No & \\
\hline \multirow{2}{*}{0.000} & 27 & 53 & Yes & \multirow{2}{*}{$\begin{array}{c}\text { Patient } \\
\text { Satisfaction }\end{array}$} \\
\hline & 52 & 26 & No & \\
\hline
\end{tabular}


Table 3. The effects of drugs (Diclofenac or placebo) on patients' mean heart rate and blood pressures

\begin{tabular}{|c|c|c|c|}
\hline \multirow{2}{*}{ Variables } & \multicolumn{2}{|c|}{ Means of three times \pm SD } & Multivariate Test \\
\cline { 2 - 4 } & $\begin{array}{c}\text { Diclofenac } \\
\text { Suppository }\end{array}$ & Placebo & P-Value \\
\hline Heart Rate & $69.88 \pm 1.09$ & $73.60 \pm 1.60$ & 0.264 \\
\hline Systolic Blood Pressure & $120.75 \pm 1.35$ & $124.41 \pm 1.33$ & 0.801 \\
\hline Diastolic Blood Pressure & $77.82 \pm 0.404$ & $78.55 \pm 0.399$ & 0.073 \\
\hline
\end{tabular}

various lithotripsy methods such as extracorporeal shockwave lithotripsy (ESWL), Trans-urethral Lithotripsy, and percutaneous Nephrolithotripsy, major steps have been taken in treatment of urinary tract stones. The present research seeks to study the effectiveness of using diclofenac suppository in order to control pain and reduce need for venous drugs during ESWL procedure in Shahid Hasheminezhad specialized center in 2014. ESWL is one of the major therapeutic methods. This method has experienced a significant rise in application due to its minor side effects. The prevalence of Urolithiasis in whole life of people has been estimated around 1 to $15 \%$ varying based upon age, gender and geographical location. The prevalence rate of this disease among men is 2 to 3 times more than in women. ESWL is the primary therapeutic method for most types of kidney stones. ${ }^{13}$ Due to its noninvasiveness, no need of general anesthesia and minor side effects, ESWL is a popular therapeutic measure in most cases of urinary tract stones with diameters smaller than $20 \mathrm{~mm} .^{14,15}$. This method makes the hospitalization and convalescence period shorter and reduces postoperation complications. Access to this non-invasive technology has extended caring for patients suffering with urinary tract stone from operation room and hospitals to clinics and outpatient centers. ${ }^{16,17}$. Based on previous results, ESWL is not without complications. ${ }^{18}$ Operators should keep in mind that timely diagnosis of clinical symptoms helps us in quick treatment of complications and problems related to lithotripsy. ${ }^{19}$ Complications include sever pain during operation, kidney complications (bleeding around kidneys, high blood pressure, reduced renal performance, and higher possibility of kidney stone recurrence), extra-renal problems such as erosions of stomach and duodenum, acute pancreatitis, lung damage if exposed to shockwaves, and hematochezia as a result of damages of the colon mucosa, ${ }^{4}$ and cardiac arrhythmias ${ }^{20,21}$ However, patients mostly complain about pain and the majority of the previous studies have been conducted on this complication. In a research by Saita et al on patients with kidney stone and upper ureter stone with a diameter of less than 15-20 mm, one group had received Ketorolac and intramuscular tramadol with betamethasone. The second group, however, had received dermal anesthetic gel containing lidocaine $1 \%$. The second group experienced less pain and they were more satisfied with ESWL. These researchers suggested a mixture of local anesthetics along with injected drugs to control pain in ESWL. ${ }^{22}$ In another research by Han et al, the analgesic effects of 30 milligrams of oral morphine were compared against 100 milligrams oral Pentazocine among patients undergoing ESWL. All patients were given 1 milligram oral lorazepam. Their research showed that mixing Pentazocine and Lorazepam creates a proper analgesic effect without complications. ${ }^{23}$ Eryildirim et al studied the analgesic effect of EMLA local anesthetics and intramuscular Sodium Diclofenac either used separately or mixed together in 20 patients candidated for ESWL. The analgesic effect of EMLA cream is much less than intramuscular Sodium Diclofenac and their mixture has no superiority over using Sodium Diclofenac alon. ${ }^{24}$ The operators were quite satisfied of the results of the diclofenac group, since only $3.7 \%$ of the patients in this group felt severe pain. Furthermore, more than $40 \%$ of participants in the placebo group felt a severe pain, requiring venous drugs. It also turned out that using diclofenac suppository had no effect on hemodynamic values of the patient. In conclusion, the use of diclofenac as a painkiller prior to ESWL plays a major role in relieving pain during the operation and the need for anesthetic drugs.

\section{List of acronyms}

ESWL-Extracorporeal shockwave lithotripsy

NSAIDs-Nonsteroidal anti-inflammatory drugs

HM3-Human Model 3

ASA-Anesthesiologists

VAS-Visual analog scale

EMLA-eutectic mixture of local anesthetics

\section{Author's contributions}

Each author contributed in equal part to the manuscript.

\section{Acknowledgments}

There are no acknowledgments.

\section{Conflict of Interest}

The authors report no conflicts of interests.

\section{Ethical Publication Statement}

We confirm that we have read the Journal's position on issues involved in ethical publication and affirm that this report is consistent with those guidelines. 


\section{Diclofenac for shock wave lithotripsy}

Eur J Transl Myol 28 (2): 210-214, 2018

\section{Corresponding Author}

Masoud Ghorbanlo, Cardiac Anesthesiologist, Hasheminejad Kidney Center, School of Medicine, Iran University of Medical Sciences, Tehran, Iran. Phone:+989121714952

Email: masoudghorbanlo@yahoo.com

E-mails of co-authors

Mehrdad Mesbah Kiaei:

Mahmoud Reza Mohaghegh:

Mehrdadmesbahkiaei@gmail.com mohaghegh_mr@yahoo.com

Gholamreza Movaseghi: gmovasaghi@yahoo.com

\section{References}

1. Mahnama M, Bagheri SM. Assessment of Urinary Tract Calculi with 16-MDCT: The Axial Versus Coronal Plane. Biosciences Biotechnology Research Asia. 2016;13:1555-9.

2. Foroutan M, Loloei B, Irvani S, Azargashb E. Accuracy of rapid urease test in diagnosing Helicobacter pylori infection in patients using NSAIDs. Saudi J Gastroenterol 2010;16:110-2.

3. Junuzovic D, Prstojevic JK, Hasanbegovic M, Lepara Z. Evaluation of Extracorporeal Shock Wave Lithotripsy (ESWL): Efficacy in Treatment of Urinary System Stones. Acta Inform Med 2014;22:309-14.

4. McDougal WS, Wein AJ, Kavoussi LR, et al. Campbell-Walsh Urology 10th Edition Review: Elsevier Health Sciences; 2011.

5. Matlaga BR. Contemporary surgical management of upper urinary tract calculi. J Urol 2009;181:2152-6.

6. Young HH, Frontz WA, Baldwin JC. Congenital obstruction of the posterior urethra . The Journal of urology 2002;167:265-7.

7. Turna B, Akbay K, Ekren F, et al. Comparative study of extracorporeal shock wave lithotripsy outcomes for proximal and distal ureteric stones. International urology and nephrology 2008;40:239.

8. El-faqih S, Husain I, Ekman P, Sharma N, Chakrabarty A, Talic R. Primary choice of intervention for distal ureteric stone: ureteroscopy or ESWL? British journal of urology 1988;62:138.

9. Walsh PC, Marschke P, Ricker D, Burnett AL. Patient-reported urinary continence and sexual function after anatomic radical prostatectomy. Urology 2000;55:58-61.

10. Zogović J. [Extracorporeal shock-wave lithotripsy in patients with manifestations of urinary tract infection]. Srpski arhiv za celokupno lekarstvo. 1996;125:285-90.

11. Alzimaity M, MOSLI H, Farsi H. Second generation extracorporeal shock wave lithotripsy treatment for childhood urolithiasis. Saudi medical journal 1992;13:54-6.

12. Saiko Y, Hirose T, Yoshida M, Saito I. [The effect of extracorporeal shock wave lithotripsy on kidney and adjacent tissue detected by magnetic resonance imaging]. Hinyokika kiyo Acta urologica Japonica 1995;41:577-80.

13. Tanagho EA. Anatomy of the lower urinary tract. Campbell's Urology 1992;1:40-69.

14. Baskin LS, Floth A, Stoller ML. Monitored anesthesia care with the standard Dornier HM3 lithotripter. J Endourol 1990;4:49-53.

15. McAninch JW, Tanagho EA. Smith's general urology: McGraw-Hill Companies, Incorporated; 2008.

16. Karlowicz KA. Urologic nursing: Principles and practice: WB Saunders Company; 1995.

17. Parker-Cohen PD. Extracorporeal shock-wave lithotripsy treatment for kidney stones. The Nurse Practitioner 1988;13:32-43.

18. Chen TP, Hwang TI, Yu CT, et al. Analgesic effect of tramadol HCL in ESWL. Zhonghua Yi Xue Za Zhi (Taipei) 1993;51:134-7.

19. Resit-Goren M, Dirim A, Ilteris-Tekin M, Ozkardes H. Time to stone clearance for ureteral stones treated with extracorporeal shock wave lithotripsy. Urology 2011;78:26-30.

20. Ganem JP, Carson CC. Cardiac arrhythmias with external fixed-rate signal generators in shock wave lithotripsy with the Medstone lithotripter. Urology 1998;51:548-52.

21. Zanetti G, Ostini F, Montanari E, et al. Cardiac dysrhythmias induced by extracorporeal shockwave lithotripsy. J Endourol 1999;13:40912.

22. Saita A, Bonaccorsi A, Aquilino M, Guzzardi F, Lazzara A, Motta M. ESWL: Comparing Two Analgesic Techniques. Urologia internationalis 2004;72:46-7.

23. Han YY, Lu HC, Tsai HJ, Hseu SS, Chan KH, Tsai SK. The analgesic effect of oral morphine or pentazocine for extracorporeal shock wave lithotripsy. Acta anaesthesiologica Sinica 2003;41:27-32.

24. Eryıldırım B, Kuyumcuoğlu U, Tarhan F, Faydacı G, Uruç F. Comparison of three analgesic treatment protocols for pain management during extracorporeal shock wave lithotripsy. Urologia internationalis 2009;82:276-9.

Submission: 16/02/18

Revisions received: 22/02/18

Acceptance: $24 / 02 / 18$ 